\title{
Multimorbidity and COPD Medication Receipt Among Medicaid Beneficiaries With Newly Diagnosed COPD
}

\author{
Mayank Ajmera MSc PhD, Usha Sambamoorthi $\mathrm{PhD}$, Aaron Metzger $\mathrm{PhD}$, \\ Nilanjana Dwibedi PhD, George Rust MD MPH, and Cindy Tworek PhD
}

\begin{abstract}
BACKGROUND: Multimorbidity is highly prevalent among patients with COPD. The association between multimorbidity and COPD medication management is not well researched. The aim of this study was to examine the association between multimorbidity and COPD medication receipt among Medicaid beneficiaries with newly diagnosed COPD. METHODS: A retrospective longitudinal dynamic cohort design was used, and data were extracted from Medicaid Analytic eXtract files from 2005 to 2008 . Medicaid beneficiaries with newly diagnosed COPD $(N=19,060)$ were identified using the International Classification of Diseases, 9th Revision, Clinical Modification, for COPD. This code (for commonly co-occurring conditions with COPD) was used to create a multimorbidity variable. These conditions included anxiety, arthritis, bipolar disorder, cardiovascular diseases, depression, diabetes, hypertension, hyperlipidemia osteoporosis, and schizophrenia. Medicaid beneficiaries with newly diagnosed COPD were categorized as: (1) physical multimorbidity only, (2) psychiatric multimorbidity only, (3) both physical and psychiatric multimorbidity, and (4) no multimorbidity. Receipt of COPD medications (short- or long-acting bronchodilators, inhaled corticosteroids) was identified using National Drug Codes. Bivariate relationships between multimorbidity and COPD medication receipt were tested using the chi-square test of independence. The associations between multimorbidity and COPD medication receipt were analyzed with logistic and multinomial logistic regression analyses. RESULTS: Among Medicaid beneficiaries with newly diagnosed COPD, 81.9\% had at least one co-occurring chronic condition. After controlling for subject characteristics, adults with multimorbidity were less likely to receive COPD medications compared with those without any inflammation-related multimorbidity. For example, those with physical multimorbidity were less likely to receive short-acting bronchodilators (adjusted odds ratio [OR] 0.76, 95\% CI 0.69-0.83), long-acting bronchodilators (adjusted OR 0.84, 95\% CI 0.76-0.92), and inhaled corticosteroids (adjusted OR 0.75, 95\% CI 0.68-0.82) compared with those with no inflammation-related multimorbidity.CONCLUSIONS: The prevalence of multimorbidity is very high among Medicaid beneficiaries with newly diagnosed COPD. Our findings indicate poor COPD medication management among those with multimorbidity. Key words: multimorbidity; COPD; medication management; Medicaid. [Respir Care 2015;60(11):1592-1602. () 2015 Daedalus Enterprises]
\end{abstract}

Introduction

Multimorbidity is defined as the coexistence of 2 or more chronic conditions. ${ }^{1-3}$ The high prevalence of mul-

Dr Ajmera is affiliated with RTI Health Solutions, Research Triangle Park, North Carolina. Drs Sambamoorthi, Dwibedi, and Tworek are affiliated with the Department of Pharmaceutical Systems and Policy, and Dr Metzger is affiliated with the Department of Psychology, School of Pharmacy, West Virginia University, Morgantown, West Virginia. Dr timorbidity has profound implications for the patient, payers, caregivers, and society. ${ }^{4}$ Multimorbidity serves as a

\footnotetext{
Rust is affiliated with the Department of Family Medicine, Morehouse School of Medicine, Atlanta, Georgia

The authors have disclosed no conflicts of interest.

Correspondence: Mayank Ajmera MSc PhD, RTI Health Solutions, 300 Park Offices Drive, Research Triangle Park, NC 27709. E-mail: majmera@rti.org.
}

DOI: $10.4187 /$ respcare. 03788 
prototype example of the Agency for Healthcare Research and Quality's definition of a complex patient, who has "two or more chronic conditions where each condition may influence the care of the other condition(s) through limitations of life expectancy, interactions between drug therapies, difficulties in establishing adequate care coordination, and/or direct contraindications to therapy for one condition by other conditions themselves." 5 Multimorbidity is a major challenge among elderly patients $\geq 65$ y old. ${ }^{6}$ However, multimorbidity is no longer being considered as just an older person's issue due to its high prevalence among younger adults $<65$ y old. 7,8

Multimorbidity is highly prevalent in patients with COPD. Using data from the National Health and Nutrition Examination Survey (1999-2008), it has been reported that adults ( $\geq 45 \mathrm{y}$ old) with COPD had significantly higher rates of coexisting arthritis $(54.6 \%$ vs $36.9 \%)$, diabetes ( $16.3 \%$ vs $12.8 \%$ ), depression ( $20.6 \%$ vs $12.5 \%$ ), osteoporosis $(16.9 \%$ vs $8.5 \%)$, coronary heart disease $(12.7 \%$ vs $6.1 \%)$, and congestive heart failure $(12.1 \%$ vs $3.9 \%)$ compared with those without COPD. ${ }^{9}$

COPD is often managed with pharmacologic agents. According to the Global Initiative for Chronic Obstructive Lung Disease (GOLD), many medications are approved for effective management of COPD symptoms and exacerbations. These include bronchodilators such as anticholinergics and $\beta$ agonists, inhaled corticosteroids, theophylline, and other medications such as phosphodiesterase-4 inhibitors and antibiotics. ${ }^{10,11}$ Existing evidence suggests that the presence of multimorbidity among adults with COPD may complicate overall COPD management due to interactions with therapies for other commonly co-occurring chronic conditions. ${ }^{12}$ For example, $\beta$ blockers, which are used to treat cardiovascular conditions, may interact with $\beta$ agonists, which are the main pharmacologic therapy for COPD management. ${ }^{13}$ Similarly, corticosteroids used to treat patients with COPD have been linked to impairment in the glucose metabolism process and thus may affect diabetes outcomes in patients with COPD and diabetes. ${ }^{14,15}$ The presence of cardiovascular disease can affect the metabolism of COPD medications. For example, theophylline clearance is reduced in patients with COPD and cardiovascular disease and may lead to poor COPDspecific outcomes in those with cardiovascular disease and COPD. ${ }^{16}$

Although multimorbidity is highly prevalent among younger adults with COPD, only a handful of studies have examined the impact of multimorbidity on health outcomes. Specifically, no study has examined the relationship between multimorbidity and COPD medication receipt in young adults (40-64 y of age) with COPD. Therefore, for the purpose of this analysis, we used administrative claims data for Medicaid enrollees, as Medicaid is an important payer of a major portion of the young adult population in

\section{QUICK LOOK}

\section{Current knowledge}

Multimorbidity is defined as the coexistence of 2 or more chronic conditions. The high prevalence of multimorbidity has profound implications for the patient, payers, caregivers, and society. Multimorbidity is highly prevalent among patients with COPD. It has been reported that adults ( $>45 \mathrm{y}$ of age) with COPD have significantly higher rates of coexisting arthritis, diabetes, depression, osteoporosis, coronary heart disease, and congestive heart failure compared with those without COPD.

\section{What this paper contributes to our knowledge}

The prevalence of multimorbidity was very high in Medicaid beneficiaries with newly diagnosed COPD. Medicaid beneficiaries with newly diagnosed COPD and multimorbidity were less likely to receive COPD medications, including bronchodilators and inhaled corticosteroids. Medication management in patients with newly diagnosed COPD is suboptimal and requires process changes to improve patient treatment.

the United States. ${ }^{17}$ Moreover, beneficiaries enrolled in the Medicaid program represent the lower socioeconomic stratum of the society. Prior evidence suggests that subjects with lower socioeconomic status have high rates of multimorbidity. ${ }^{6,18}$ In addition, multimorbidity has a worse impact on patients in the lower socioeconomic stratum of society compared with higher socioeconomic groups. ${ }^{19}$

Therefore, the primary objectives of this study were to estimate the prevalence of commonly co-occurring chronic conditions (multimorbidity) in Medicaid beneficiaries with newly diagnosed COPD and also to examine the association between multimorbidity and receipt of common medications required to treat patients with COPD using multiyear and multistate Medicaid claims data. We hypothesized that due to competing demands associated with management of multimorbidity, the presence of multimorbidity would be associated with reduced COPD medication receipt.

\section{Methods}

\section{Study Design}

We used a retrospective longitudinal dynamic cohort design and extracted data from Medicaid Analytic eXtract files from 2005 to 2008. We identified Medicaid beneficiaries with diagnosed COPD in 2006 and 2007. The first- 
service date with COPD diagnosis was recorded as the index date. The procedure used to identify the study population is described in detail below. The two distinct study observation periods were the baseline period (defined as $1 \mathrm{y}$ before the index date) and the follow-up period (defined as $1 \mathrm{y}$ after the index date). The baseline period was used to ascertain the presence of multimorbidity and other subject characteristics, whereas the follow-up period was used to measure the receipt of COPD medications.

\section{Data Source}

Medicaid Analytic eXtract files containing information on enrollment, inpatient visits, other therapy, and prescription drug utilization were used. The personal summary file provided information on eligibility, demographics (age, sex, and race), managed care enrollment, and utilization summary. The other 4 files contained information on feefor-service claims for services used. Other therapy files included information regarding claims for Medicaid services provided at an out-patient level, such as clinic, physician, laboratory, and home-health services. Hospitalization diagnosis codes, procedures, hospitalization stay, and expenditures related to hospitalizations were extracted from the in-patient files. National Drug Codes for prescription drugs, supply days, fill dates, and payments for prescription drugs were obtained from the prescription drug files.

We used data for beneficiaries residing in the following states: California, Illinois, New York, and Texas. These states were chosen to capture the diverse geographic and racial/ethnic populations represented by Medicaid. We received approval from the institutional review board of West Virginia University for analysis of Medicaid Analytic eXtract data to conduct this study.

\section{Area Resource File}

We also determined each Medicaid beneficiary's contextual county-level variables using the Area Resource File. The Area Resource File is a comprehensive county-level data set compiled by the Bureau of Health Professions of the Health Resources Service Administration. The Area Resource File contains $>6,000$ variables providing information about the nation's counties. The information contained within the Area Resource File includes the type of health facilities in the various counties, number and type of health professions, resource scarcity measures, health status, economic activity, health-training programs, and socioeconomic and environmental characteristics. These files also include county codes and descriptors that allow linkage with several secondary data sets, such as Medicaid Analytic eXtract. We used the county codes and state information to link Medicaid Analytic eXtract files with the 2005 Area Resource File.

\section{Study Population: Medicaid Beneficiaries With Newly Diagnosed COPD}

Medicaid beneficiaries with diagnosed COPD were identified using in- and out-patient visits (physician office visits and clinic services only). Medicaid beneficiaries with at least one in-patient visit or 2 out-patient visits $14 \mathrm{~d}$ apart (obtained using the type of service codes) for COPD based on International Classification of Diseases, Ninth Revision, Clinical Modification (ICD-9-CM), codes for chronic bronchitis (491.xx), emphysema (492.xx), or unspecified chronic airway obstruction (496.xx) were considered to have diagnosed COPD. These diagnosis codes have been used in prior published research (codes 491.xx, 492.xx and 496.xx) to identify COPD and to evaluate medical treatment and health outcomes in subjects with COPD. ${ }^{20,21}$ Indeed, the sensitivity and specificity of using ICD-9-CM codes to identify subjects with COPD has been established. ${ }^{22,23}$ Gershon et al ${ }^{23}$ reported that identifying COPD using one or more ambulatory claims and/or one or more hospitalizations for COPD resulted in a sensitivity of $85.0 \%$ (95\% CI 77.0-91.0) and a specificity of $78.4 \%$ (95\% CI 73.6-82.7). However, we used one in-patient claim or at least 2 out-patient claims to identify subjects with COPD to increase the specificity of the algorithm. We included only Medicaid beneficiaries with newly diagnosed COPD. To obtain subjects with newly diagnosed COPD, we created a washout period ( $1 \mathrm{y}$ before the index date of COPD diagnosis). Only Medicaid beneficiaries who did not have a COPD diagnosis in the washout period were considered to have newly diagnosed COPD and were included in our study population.

Other inclusion criteria included: 40-64 y of age (among non-elderly population, this age group is at the highest risk for COPD), continuous eligibility during the baseline and follow-up periods, no dual Medicaid/Medicare coverage (dual eligibility would represent high cost and severe beneficiaries), enrolled in fee-for-service plans throughout the study observation period, alive during the study observation period, and use of services (in- or out-patient).

\section{Dependent Variables: COPD Medication Receipt}

The common pharmacologic treatment for patients with COPD is the administration of maintenance bronchodilators and inhaled corticosteroids. ${ }^{10,11}$ COPD medications were identified using the National Drug Codes obtained from various publicly available sources, such as the FDA, Multum Lexicon National Drug Codes from the Agency for Healthcare Research and Quality Medical Expenditure Panel Survey, National Committee for Quality Assurance database, and Department of Veterans Affairs. We identified the following binary dependent variables for COPD medication receipt: (1) receipt of short-acting bronchodi- 
lators, including $\beta$ agonists and inhaled anticholinergics (yes/no); (2) receipt of long-acting bronchodilators, including $\beta$ agonists and inhaled anticholinergics (yes/no); and (3) receipt of inhaled corticosteroids (yes/no). Furthermore, we also created an aggregate variable with 4 categories for receipt of type of bronchodilator: (1) receipt of short-acting bronchodilators only, (2) receipt of long-acting bronchodilators only, (3) receipt of both types of bronchodilator, and (4) no bronchodilator use.

\section{Key Independent Variable: Multimorbidity}

When examining multimorbidity in patients with COPD, it is important to note that COPD is characterized mainly as an inflammatory disease that is associated with progressive and persistent air-flow limitation. ${ }^{24}$ It has been suggested that there may be a high prevalence of inflammation-related chronic conditions in patients with COPD due to elevated levels of inflammatory mediators. Although the exact mechanisms have not yet been established, researchers suggest that either inflammation in COPD may have a spillover effect, or the inflammation may be part of systemic inflammation. ${ }^{25}$ A review on systemic manifestations of COPD suggested that systemic inflammation in COPD may lead to various inflammation-related chronic diseases, such as arthritis, cardiovascular diseases, depression, diabetes, and osteoporosis. ${ }^{26,27}$ Indeed, researchers have suggested that COPD should be considered as part of the chronic systemic inflammatory syndrome. ${ }^{26}$ Therefore, to construct a multimorbidity variable in subjects with COPD, we selected chronic inflammatory conditions that commonly co-occur with COPD. ${ }^{9}$ Our selection of conditions was also guided by Department of Health and $\mathrm{Hu}-$ man Services priorities for research, policy, program, and practice. ${ }^{28}$

We created binary indicator variables for the presence of commonly co-occurring chronic conditions with COPD using the ICD-9-CM codes obtained from the Agency for Healthcare Research and Quality Clinical Classifications Software. Chronic physical conditions that were used to create the multimorbidity variable included arthritis, cardiovascular disease, diabetes, hypertension, hyperlipidemia, and osteoporosis. Chronic psychiatric conditions included anxiety, bipolar disorder, depression, and schizophrenia. These conditions were selected because of their common co-occurrence with COPD possibly due to common inflammation-related pathways and shared risk factors, such as tobacco smoking. ${ }^{9}$ Any Medicaid beneficiary with newly diagnosed COPD who had one in-patient visit or at least 2 out-patient visits for these conditions during the baseline period were considered as having the chronic condition. We further operationalized the multimorbidity variable into 4 groups: (1) physical multimorbidity only, (2) psychiatric multimorbidity only, (3) both physical and psychiatric multimorbidity, and (4) no multimorbidity.

\section{Other Independent Variables}

As the relationship between multimorbidity and medication receipt is generally confounded by many external factors, we controlled for a comprehensive set of covariates based on the Andersen health-care utilization model. ${ }^{29}$ This conceptual framework has been extensively used in prior studies to understand the use of health services. ${ }^{30}$ The Andersen health-care utilization model suggests that use of health services is associated with a patient's predisposing characteristics (eg, age, sex), enabling resources (eg, socioeconomic status, environmental factors), and need characteristics (eg, clinical conditions). On the basis of these characteristics, we collected information on the following variables, which may influence the relationship between multimorbidity and COPD medication receipt.

Predisposing or Subject Characteristics. These variables included demographic characteristics such as sex, race (white, African-American, other), and age (40-49, 50-59, 60-64 y). Enabling characteristics included Medicaid eligibility due to income (yes/no) and medical needs (yes/no). Need characteristics included number of other clinical conditions (none, 1-3, 4-6, >6), alcohol abuse (yes/no), substance abuse (yes/no), tobacco use (yes/no), and polypharmacy ( $<10$ drug classes, $\geq 10$ drug classes). Each state administering Medicaid coverage to eligible beneficiaries has different policies; for example, states have restrictions on medications that can be covered. To account for policy differences, we included a dummy variable for the states (California, Illinois, New York, and Texas) in our analyses.

External Environment or County-Level Characteristics. These variables included above high-school education density (quartiles), unemployment density (quartiles), poverty density (quartiles), metro status (metro/non-metro), primary care provider shortage (yes/no), mental health shortage (yes/no), primary care provider density (quartiles), specialist provider density (quartiles), hospital bed density (quartiles), rural health clinics (yes/no), psychiatric hospital (yes/no), pulmonologist density (high [ie, above 75th percentile], low [ie, below 75th percentile]), and cardiologist density (high [ie, above 75th percentile], low [ie, below 75th percentile]). In the results section, we primarily highlight the relationship between multimorbidity and medication receipt after controlling for other independent variables that may influence medication receipt. 


\section{Statistical Analyses}

Bivariate Analyses. Chi-square tests of independence were used to determine subgroup differences in the presence of multimorbidity and receipt of COPD medications in subjects with newly diagnosed COPD. We also present the results from unadjusted analyses with odds ratios (ORs), 95\% CIs, and levels of statistical significance.

Multivariate Analyses. Binary and multinomial logistic regression analyses were used to examine the relationship between multimorbidity and COPD medication receipt. Parameter estimates are represented as adjusted OR with 95\% CI. All analyses were conducted using SAS 9.3 (SAS Institute, Cary, North Carolina).

\section{Results}

The final study population consisted of 19,060 Medicaid beneficiaries $(40-64$ y old) with newly diagnosed COPD. The algorithm used to define the study cohort is shown in Figure 1. The majority of the Medicaid beneficiaries with newly diagnosed COPD were women $(59.5 \%)$, white $(48.6 \%), 50-59$ y old $(48.4 \%)$, and residents of California (54.5\%).

\section{Prevalence of Multimorbidity}

Overall, among Medicaid beneficiaries with newly diagnosed COPD $(n=19,060), 36.3 \%$ had physical multimorbidity, $13.5 \%$ had psychiatric multimorbidity, $32.1 \%$ had both, and $18.1 \%$ did not have any multimorbidity. With regard to chronic conditions, $37.8 \%$ of subjects had hypertension, 35.8\% had musculoskeletal disorders, $26.3 \%$ had depression, $19.8 \%$ had diabetes, $18.5 \%$ had schizophrenia, $17.9 \%$ had cardiovascular conditions, $12.3 \%$ had hyperlipidemia, $12.7 \%$ had anxiety, and $11.4 \%$ had bipolar disorder. A detailed description of study population characteristics by the presence of multimorbidity is presented in Table 1.

\section{COPD Medication Receipt}

Findings from the bivariate and multivariate analyses on COPD medication receipt with multimorbidity as the key independent variable are depicted in Table 2. Approximately $64 \%$ of Medicaid beneficiaries with newly diagnosed COPD had at least one prescription for short-acting bronchodilators during the follow-up period. The percentages of Medicaid beneficiaries with long-acting bronchodilators and inhaled corticosteroids were $39.2 \%$ and $43.3 \%$, respectively. Results from the unadjusted logistic regression analyses of COPD medication receipt revealed that Medicaid beneficiaries with mental illness were less likely

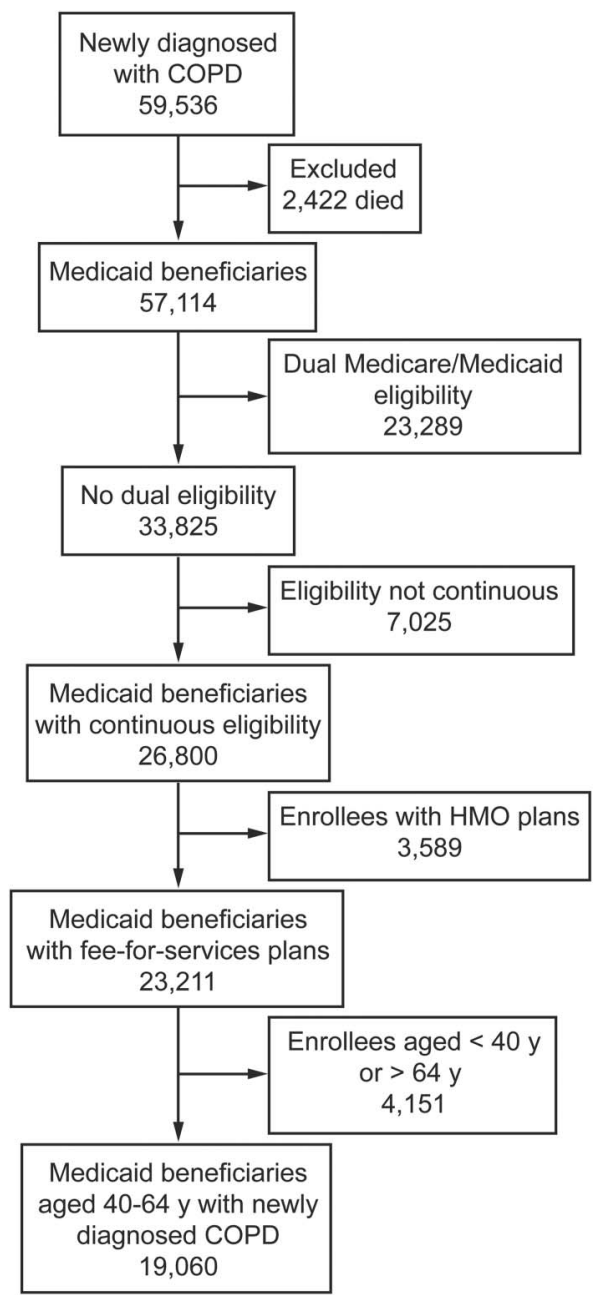

Fig. 1. Flow chart. $\mathrm{HMO}=$ health maintenance organization.

to receive short-acting bronchodilators (OR $0.69,95 \%$ CI 0.62-0.77), long-acting bronchodilators (OR 0.73, 95\% CI 0.66-0.82), and inhaled corticosteroids (OR 0.73, 95\% CI $0.66-0.81)$. Similarly, those with both physical and psychiatric chronic conditions were less likely to receive short- and long-acting bronchodilators and inhaled corticosteroids.

Findings from the multivariate logistic regression analyses (see Table 2) of COPD medication receipt in Medicaid beneficiaries with newly diagnosed COPD revealed that those with any multimorbidity (ie, physical conditions, serious mental illness, or both) were significantly less likely to receive COPD medications. For example, those with physical conditions only were less likely to receive short-acting bronchodilators (adjusted OR 0.76, 95\% CI 0.79-0.83), long-acting bronchodilators (adjusted OR $0.84,95 \%$ CI $0.76-0.92$ ), and inhaled corticosteroids (adjusted OR 0.75, 95\% CI 0.68-0.82) compared with those with no multimorbidity. 


\section{Multimorbidity and COPD Medication ReCEIPT}

Table 1. Description of Study Population Characteristics by Multimorbidity Categories Among Medicaid Beneficiaries With Newly Diagnosed COPD

\begin{tabular}{|c|c|c|c|c|c|c|c|c|c|}
\hline \multirow{2}{*}{ Characteristic } & \multicolumn{2}{|c|}{ Physical Only } & \multicolumn{2}{|c|}{ Psychiatric Only } & \multicolumn{2}{|c|}{ Both } & \multicolumn{2}{|c|}{ None } & \multirow{2}{*}{$P$} \\
\hline & $n$ & Row $\%$ & $n$ & Row $\%$ & $n$ & Row \% & $n$ & Row $\%$ & \\
\hline Total & 6,919 & 36.3 & 2,572 & 13.5 & 6,121 & 32.1 & 3,448 & 18.1 & \\
\hline \multicolumn{10}{|l|}{ Cohort } \\
\hline 2005-2007 & 3,841 & 35.8 & 1,502 & 14.0 & 3,448 & 32.2 & 1,930 & 18.0 & \\
\hline 2006-2008 & 3,078 & 36.9 & 1,070 & 12.8 & 2,673 & 32.1 & 1,518 & 18.2 & \\
\hline Sex & & & & & & & & & $<.001$ \\
\hline Females & 3,998 & 35.3 & 1,478 & 13.0 & 4,009 & 35.4 & 1,849 & 16.3 & \\
\hline Males & 2,921 & 37.8 & 1,094 & 14.2 & 2,112 & 27.3 & 1,599 & 20.7 & \\
\hline Race/ethnicity & & & & & & & & & $<.001$ \\
\hline White & 2,990 & 32.2 & 1,419 & 15.3 & 3,134 & 33.8 & 1,729 & 18.6 & \\
\hline African-American & 2,127 & 41.8 & 492 & 9.7 & 1,584 & 31.2 & 882 & 17.3 & \\
\hline Others & 1,802 & 38.3 & 661 & 14.1 & 1,403 & 29.8 & 837 & 17.8 & \\
\hline Age, y & & & & & & & & & $<.001$ \\
\hline $40-49$ & 1,650 & 26.0 & 1,216 & 19.2 & 2,249 & 35.5 & 1,229 & 19.4 & \\
\hline $50-59$ & 3,578 & 38.7 & 1,094 & 11.8 & 2,966 & 32.1 & 1,596 & 17.3 & \\
\hline $60-64$ & 1,691 & 48.6 & 262 & 7.5 & 906 & 26.0 & 623 & 17.9 & \\
\hline State & & & & & & & & & $<.001$ \\
\hline California & 3,927 & 37.8 & 1,430 & 13.8 & 3,218 & 31.0 & 1,822 & 17.5 & \\
\hline Illinois & 1,478 & 36.0 & 504 & 12.3 & 1,471 & 35.8 & 657 & 16.0 & \\
\hline New York & 685 & 26.2 & 450 & 17.2 & 911 & 34.8 & 569 & 21.8 & \\
\hline Texas & 829 & 42.8 & 188 & 9.7 & 521 & 26.9 & 400 & 20.6 & \\
\hline Poverty eligibility & & & & & & & & & $<.001$ \\
\hline Yes & 6,380 & 36.5 & 2,387 & 13.7 & 5,637 & 32.3 & 3,072 & 17.6 & \\
\hline No & 539 & 34.0 & 185 & 11.7 & 484 & 30.6 & 376 & 23.7 & \\
\hline Medical eligibility & & & & & & & & & $<.001$ \\
\hline Yes & 590 & 28.9 & 290 & 14.2 & 780 & 38.2 & 382 & 18.7 & \\
\hline No & 6,329 & 37.2 & 2,282 & 13.4 & 5,341 & 31.4 & 3,066 & 18.0 & \\
\hline No. of clinical conditions & & & & & & & & & $<.001$ \\
\hline None & 97 & 20.5 & 94 & 19.9 & 58 & 12.3 & 224 & 47.4 & \\
\hline $1-3$ & 1,078 & 30.4 & 655 & 18.5 & 605 & 17.1 & 1,210 & 34.1 & \\
\hline $4-6$ & 1,668 & 37.3 & 727 & 16.3 & 1,114 & 24.9 & 964 & 21.6 & \\
\hline$>6$ & 4,076 & 38.6 & 1,096 & 10.4 & 4,344 & 41.1 & 1,050 & 9.9 & \\
\hline Alcohol abuse & & & & & & & & & $<.001$ \\
\hline Yes & 436 & 23.9 & 317 & 17.4 & 845 & 46.3 & 226 & 12.4 & \\
\hline No & 6,483 & 37.6 & 2,255 & 13.1 & 5,276 & 30.6 & 3,222 & 18.7 & \\
\hline Substance abuse & & & & & & & & & $<.001$ \\
\hline Yes & 581 & 22.0 & 483 & 18.3 & 1,241 & 47.0 & 335 & 12.7 & \\
\hline No & 6,338 & 38.6 & 2,089 & 12.7 & 4,880 & 29.7 & 3,113 & 19.0 & \\
\hline Tobacco Use & & & & & & & & & $<.001$ \\
\hline Yes & 723 & 28.5 & 366 & 14.4 & 1,147 & 45.2 & 302 & 11.9 & \\
\hline No & 6,196 & 37.5 & 2,206 & 13.4 & 4,974 & 30.1 & 3,146 & 19.0 & \\
\hline Polypharmacy & & & & & & & & & $<.001$ \\
\hline Yes & 3,984 & 40.4 & 897 & 9.1 & 4,189 & 42.5 & 793 & 8.0 & \\
\hline No & 2,935 & 31.9 & 1,675 & 18.2 & 1,932 & 21.0 & 2,655 & 28.9 & \\
\hline Area Resource File county-level variables & & & & & & & & & $<.001$ \\
\hline Above high-school education density & & & & & & & & & \\
\hline Q1 & 3,663 & 36.4 & 1,359 & 13.5 & 3,424 & 34.1 & 1,609 & 16.0 & \\
\hline Q2 & 1,056 & 38.4 & 297 & 10.8 & 828 & 30.1 & 567 & 20.6 & \\
\hline Q3 & 1,364 & 35.5 & 564 & 14.7 & 1,152 & 30.0 & 763 & 19.9 & \\
\hline Q4 & 836 & 34.6 & 352 & 14.6 & 717 & 29.7 & 509 & 21.1 & \\
\hline
\end{tabular}




\section{Multimorbidity ANd COPD MedicAtion Receipt}

Table 1. Continued

\begin{tabular}{|c|c|c|c|c|c|c|c|c|c|}
\hline \multirow{2}{*}{ Characteristic } & \multicolumn{2}{|c|}{ Physical Only } & \multicolumn{2}{|c|}{ Psychiatric Only } & \multicolumn{2}{|c|}{ Both } & \multicolumn{2}{|c|}{ None } & \multirow{2}{*}{$P$} \\
\hline & $n$ & Row $\%$ & $n$ & Row \% & $n$ & Row \% & $n$ & Row \% & \\
\hline Unemployment density & & & & & & & & & $<.001$ \\
\hline Q1 & 5,082 & 35.9 & 2,015 & 14.2 & 4,682 & 33.1 & 2,376 & 16.8 & \\
\hline Q2 & 961 & 37.4 & 295 & 11.5 & 746 & 29.0 & 568 & 22.1 & \\
\hline Q3 & 674 & 38.0 & 202 & 11.4 & 531 & 29.9 & 368 & 20.7 & \\
\hline Q4 & 202 & 36.1 & 60 & 10.7 & 162 & 28.9 & 136 & 24.3 & \\
\hline Poverty density & & & & & & & & & $<.001$ \\
\hline Q1 & 537 & 33.9 & 271 & 17.1 & 476 & 30.1 & 299 & 18.9 & \\
\hline Q2 & 975 & 36.2 & 370 & 13.7 & 785 & 29.1 & 564 & 20.9 & \\
\hline Q3 & 1,312 & 37.4 & 434 & 12.4 & 1,014 & 28.9 & 748 & 21.3 & \\
\hline Q4 & 4,095 & 36.3 & 1,497 & 13.3 & 3,846 & 34.1 & 1,837 & 16.3 & \\
\hline Metro & & & & & & & & & $<.001$ \\
\hline Yes & 6,013 & 36.4 & 2,285 & 13.8 & 5,340 & 32.3 & 2,891 & 17.5 & \\
\hline No & 906 & 35.8 & 287 & 11.3 & 781 & 30.9 & 557 & 22.0 & \\
\hline Primary care provider shortage & & & & & & & & & $<.001$ \\
\hline Yes & 6,658 & 36.4 & 2,469 & 13.5 & 5,899 & 32.3 & 3,264 & 17.8 & \\
\hline No & 261 & 33.9 & 103 & 13.4 & 222 & 28.8 & 184 & 23.9 & \\
\hline Psychiatric health specialist shortage & & & & & & & & & $<.001$ \\
\hline Yes & 6,239 & 36.6 & 2,301 & 13.5 & 5,494 & 32.2 & 3,022 & 17.7 & \\
\hline No & 680 & 33.9 & 271 & 13.5 & 627 & 31.3 & 426 & 21.3 & \\
\hline Primary care provider density & & & & & & & & & $<.001$ \\
\hline Q1 & 335 & 41.7 & 75 & 9.3 & 226 & 28.1 & 168 & 20.9 & \\
\hline Q2 & 1,221 & 38.0 & 363 & 11.3 & 946 & 29.4 & 683 & 21.3 & \\
\hline Q3 & 998 & 37.0 & 340 & 12.6 & 801 & 29.7 & 559 & 20.7 & \\
\hline Q4 & 4,365 & 35.4 & 1,794 & 14.5 & 4,148 & 33.6 & 2,038 & 16.5 & \\
\hline Specialist density & & & & & & & & & $<.001$ \\
\hline Q1 & 326 & 40.9 & 71 & 8.9 & 226 & 28.4 & 174 & 21.8 & \\
\hline Q2 & 1,139 & 37.7 & 346 & 11.4 & 909 & 30.0 & 631 & 20.9 & \\
\hline Q3 & 1,089 & 37.6 & 361 & 12.5 & 838 & 29.0 & 605 & 20.9 & \\
\hline Q4 & 4,365 & 35.4 & 1,794 & 14.5 & 4,148 & 33.6 & 2,038 & 16.5 & \\
\hline Hospital bed density & & & & & & & & & $<.001$ \\
\hline Q1 & 389 & 38.9 & 112 & 11.2 & 299 & 29.9 & 200 & 20.0 & \\
\hline Q2 & 1,179 & 37.9 & 378 & 12.2 & 895 & 28.8 & 659 & 21.2 & \\
\hline Q3 & 3,934 & 36.7 & 1,451 & 13.6 & 3,555 & 33.2 & 1,765 & 16.5 & \\
\hline Q4 & 1,417 & 33.4 & 631 & 14.9 & 1,372 & 32.3 & 824 & 19.4 & \\
\hline Rural health clinic & & & & & & & & & $<.001$ \\
\hline Yes & 2,962 & 39.4 & 866 & 11.5 & 2,192 & 29.2 & 1,498 & 19.9 & \\
\hline No & 3,957 & 34.3 & 1,706 & 14.8 & 3,929 & 34.0 & 1,950 & 16.9 & \\
\hline Psychiatric hospital & & & & & & & & & $<.001$ \\
\hline Yes & 4,302 & 35.5 & 1,731 & 14.3 & 4,041 & 33.4 & 2,030 & 16.8 & \\
\hline No & 2,617 & 37.6 & 841 & 12.1 & 2,080 & 29.9 & 1,418 & 20.4 & \\
\hline Pulmonologist density & & & & & & & & & $<.001$ \\
\hline High & 4,747 & 35.5 & 1,926 & 14.4 & 4,462 & 33.3 & 2,249 & 16.8 & \\
\hline Low & 2,172 & 38.3 & 646 & 11.4 & 1,659 & 29.2 & 1,199 & 21.1 & \\
\hline Cardiologist density & & & & & & & & & $<.001$ \\
\hline High & 4,386 & 35.2 & 1,816 & 14.6 & 4,205 & 33.7 & 2,069 & 16.6 & \\
\hline Low & 2,533 & 38.5 & 756 & 11.5 & 1,916 & 29.1 & 1,379 & 20.9 & \\
\hline $\begin{array}{l}\text { are based on } 19,060 \text { Medicaid beneficiaries wit } \\
\text { imorbidity rates by beneficiary characteristics. } \\
\text { quartile }\end{array}$ & diag & D 00 & Medic & " CAUc & om 20 & 8. $P$ va & esent s & "group & ces in \\
\hline
\end{tabular}


Table 2. COPD Medication Use in Medicaid Beneficiaries With Newly Diagnosed COPD

\begin{tabular}{|c|c|c|c|c|c|c|c|c|}
\hline \multirow[t]{2}{*}{ COPD Medication } & \multirow[t]{2}{*}{$n$} & \multirow[t]{2}{*}{ Row \% } & \multicolumn{3}{|c|}{$\begin{array}{l}\text { Unadjusted Logistic Regression } \\
\text { Analysis of COPD } \\
\text { Medication Receipt }\end{array}$} & \multicolumn{3}{|c|}{$\begin{array}{l}\text { Adjusted Logistic Regression } \\
\text { Analysis of COPD } \\
\text { Medication Receipt }\end{array}$} \\
\hline & & & OR & $95 \% \mathrm{CI}$ & $P$ & Adjusted OR & $95 \% \mathrm{CI}$ & $P$ \\
\hline \multicolumn{9}{|c|}{ Short-acting bronchodilators } \\
\hline Total & 12,177 & 63.9 & & & & & & \\
\hline \multicolumn{9}{|l|}{ Multimorbidity } \\
\hline Physical only & 4,540 & 65.6 & 0.93 & $0.85-1.02$ & & 0.76 & $0.69-0.83$ & $<.001$ \\
\hline Psychiatric only & 1,506 & 58.6 & 0.69 & $0.62-0.77$ & $<.001$ & 0.61 & $0.55-0.69$ & $<.001$ \\
\hline Both & 3,814 & 62.3 & 0.81 & $0.74-0.88$ & $<.001$ & 0.54 & $0.49-0.59$ & $<.001$ \\
\hline None & 2,317 & 67.2 & & & & & & \\
\hline \multicolumn{9}{|c|}{ Long-acting bronchodilators } \\
\hline Total & 7,474 & 39.2 & & & & & & \\
\hline \multicolumn{9}{|l|}{ Multimorbidity } \\
\hline Physical only & 2,842 & 41.1 & 0.93 & $0.86-1.01$ & & 0.84 & $0.76-0.92$ & $<.001$ \\
\hline Psychiatric only & 914 & 35.5 & 0.73 & $0.66-0.82$ & $<.001$ & 0.69 & $0.62-0.77$ & $<.001$ \\
\hline Both & 2,240 & 36.6 & 0.77 & $0.71-0.84$ & $<.001$ & 0.62 & $0.56-0.68$ & $<.001$ \\
\hline None & 1,478 & 42.9 & & & & & & \\
\hline \multicolumn{9}{|l|}{ Inhaled corticosteroids } \\
\hline Total & 8,260 & 43.3 & & & & & & \\
\hline \multicolumn{9}{|l|}{ Multimorbidity } \\
\hline Physical Only & 3,091 & 44.7 & 0.96 & $0.89-1.04$ & & 0.75 & $0.68-0.82$ & $<.001$ \\
\hline Psychiatric only & 978 & 38.0 & 0.73 & $0.66-0.81$ & $<.001$ & 0.64 & $0.57-0.71$ & $<.001$ \\
\hline Both & 2,618 & 42.8 & 0.89 & $0.82-0.97$ & $<.01$ & 0.60 & $0.54-0.66$ & $<.001$ \\
\hline None & 1,573 & 45.6 & & & & & & \\
\hline \multicolumn{9}{|c|}{$\begin{array}{l}\text { Data are based on 19,060 Medicaid beneficiaries with newly diagnosed COPD obtained from Medicaid Analytic eXtract files from } 2005 \text { to } 2008 . P \text { values represent significant group differences in } \\
\text { likelihood of respective COPD medication use by multimorbidity compared with the reference group (none) obtained from unadjusted and adjusted logistic regression analyses. Adjusted logistic } \\
\text { regression analyses controlled for cohort year, sex, race, age, state, poverty eligibility, number of other clinical conditions, serious mental illness, alcohol abuse, substance abuse, tobacco use, } \\
\text { polypharmacy, and county-level variables (including above high-school education density [quartiles], unemployment density [quartiles], poverty density [quartiles], metro status, primary care shortage, } \\
\text { mental health shortage, primary care provider density [quartiles], hospital bed density [quartiles], psychiatric hospital, pulmonologist density, and cardiologist density). } \\
\text { OR = odds ratio }\end{array}$} \\
\hline
\end{tabular}

Similarly, subjects with both physical and psychiatric multimorbidity were $46 \%$ less likely to use short-acting bronchodilators (adjusted OR 0.54, 95\% CI 0.49-0.59), $38 \%$ less likely to use to long-acting bronchodilators (adjusted OR 0.62 , 95\% CI 0.56-0.68), and 40\% less likely to use inhaled corticosteroids (adjusted OR 0.60, 95\% CI 0.54-0.66) compared with those with no multimorbidity. In addition, certain subject characteristics, ecosystem-level variables, and policy-environment variables were significantly associated with COPD medication receipt.

To understand the overall bronchodilator use in Medicaid beneficiaries with newly diagnosed COPD, we created an aggregate bronchodilator-use variable with 4 categories (short-acting only, long-acting only, both, and none). The majority $(47.2 \%)$ of beneficiaries who used at least one bronchodilator ( $n=13,310)$ in the follow-up period were in the both-bronchodilator-use group. Findings from multinomial logistic regression analyses (Table 3 ) revealed that subjects in any of the multimorbidity categories were less likely to receive short-acting bronchodilators only and combined short- and long-acting bronchodilators. The presence of psychiatric multimorbidity and both physical and psychiatric multimorbidity was associated with reduced receipt of long-acting bronchodilators.

\section{Discussion}

In this study, we examined the impact of multimorbidity on COPD medication receipt in Medicaid beneficiaries with newly diagnosed COPD. In this study cohort, approximately three fourths of subjects had multimorbidity. Although no study has comprehensively examined the prevalence of all commonly occurring comorbidities in subjects with COPD, the rates for some of the subject conditions obtained from our analyses are somewhat consistent with those found in other studies that evaluated comorbidity burden in subjects with COPD. ${ }^{9,31}$

In this study, we found that after controlling for subject characteristics and county-level socioeconomic and healthcare environment, Medicaid beneficiaries with newly diagnosed COPD and multimorbidity were less likely to receive COPD medications, including bronchodilators and inhaled corticosteroids. To the best of our knowledge, this is the first study to examine the relationship between mul- 
Table 3. Multinomial Logistic Regression Analysis of Type of Bronchodilator Used in Medicaid Beneficiaries With Newly Diagnosed COPD

\begin{tabular}{|c|c|c|c|c|c|}
\hline Type of Bronchodilator & $n$ & Row $\%$ & Adjusted OR & $95 \% \mathrm{CI}$ & $P$ \\
\hline \multicolumn{6}{|c|}{ Short-acting bronchodilators only } \\
\hline Total & 5,836 & 30.6 & & & \\
\hline \multicolumn{6}{|l|}{ Multimorbidity } \\
\hline Physical only & 2,149 & 31.1 & 0.82 & $0.73,0.92$ & $<.001$ \\
\hline Psychiatric only & 731 & 28.4 & 0.66 & $0.57,0.76$ & $<.001$ \\
\hline Both & 1,928 & 31.5 & 0.61 & $0.54,0.68$ & $<.001$ \\
\hline None & 1,028 & 29.8 & & & \\
\hline \multicolumn{6}{|c|}{ Long-acting bronchodilators only } \\
\hline Total & 1,133 & 5.9 & & & \\
\hline \multicolumn{6}{|l|}{ Multimorbidity } \\
\hline Physical only & 451 & 6.5 & 1.04 & $0.85,1.27$ & \\
\hline Psychiatric only & 139 & 5.4 & 0.77 & $0.60,0.98$ & $<.05$ \\
\hline Both & 354 & 5.8 & 0.78 & $0.63,0.96$ & $<.05$ \\
\hline None & 189 & 5.5 & & & \\
\hline \multicolumn{6}{|l|}{ Both bronchodilators } \\
\hline Total & 6,341 & 33.3 & & & \\
\hline \multicolumn{6}{|l|}{ Multimorbidity } \\
\hline Physical only & 2,391 & 34.6 & 0.72 & $0.64,0.80$ & $<.001$ \\
\hline Psychiatric only & 775 & 30.1 & 0.53 & $0.46,0.61$ & $<.001$ \\
\hline Both & 1,886 & 30.8 & 0.44 & $0.39,0.49$ & $<.001$ \\
\hline None & 1,289 & 37.4 & & & \\
\hline
\end{tabular}

Data are based on 19,060 Medicaid beneficiaries with newly diagnosed COPD obtained from Medicaid Analytic eXtract files from 2005 to 2008. $P$ values represent significant group differences in likelihood of bronchodilator use by multimorbidity compared with the reference group (none) obtained from multinomial logistic regression analyses. Adjusted logistic regression analyses controlled for cohort year, sex, race, age, state, poverty eligibility, number of other clinical conditions, serious mental illness, alcohol abuse, substance abuse, tobacco use, polypharmacy, and county-level variables (including above high-school education density [quartiles], unemployment density [quartiles], poverty density [quartiles], metro status, primary care shortage, mental health shortage, primary care provider density [quartiles], hospital bed density [quartiles], psychiatric hospital, pulmonologist density, and cardiologist density).

OR $=$ odds ratio

timorbidity and COPD medication receipt in Medicaid beneficiaries with newly diagnosed COPD. Therefore, we cannot compare our findings and can only speculate reasons for lower receipt of COPD medications in those with multimorbidity. Provider and patient factors may affect COPD medication receipt. Providers may be cautious in prescribing COPD medications due to adverse effects and competing demands from other conditions. Studies have suggested that certain COPD medications are associated with adverse effects that could complicate management of allied co-occurring conditions. For example, bronchodilators such as long-acting $\beta$ agonists and inhaled anticholinergics are associated with cardiovascular complications such as unstable angina and myocardial infarction. ${ }^{32-35} \mathrm{Sim}-$ ilarly, inhaled corticosteroids may increase blood glucose levels in those with prediabetes or diabetes. ${ }^{15,36}$ Therefore, it is important to weigh the benefits and safety issues in the health-care management of patients with COPD and multimorbidity. Our findings underscore the need to develop clinical practice guidelines that are specific to patients with COPD and multimorbidity.

It is also possible that patients may not prioritize COPD management when they have multiple chronic conditions. A recently conducted qualitative study in Australia that examined the "patients' perspectives on the impact of a new COPD diagnosis in the face of multimorbidity" indicated that subjects with existing chronic conditions ascribed low importance to the management of a new COPD diagnosis. ${ }^{37}$ Moreover, subjects with COPD and multimorbidity were challenged by multiple-medication use and thus had poor COPD self-management capacity. Although this study was conducted in Australia and had a limited sample size, it provides an important understanding of the relationship between multimorbidity and COPD medication receipt.

The advantages of our study are: the use of administrative claims data, which provided us the ability to identify medical conditions using diagnosis codes; availability of prescription medication claims; examination of a high-risk and low-income population; and ability to track capture utilization over time. Our study was strengthened by linking Medicaid files with the Area Resource File to comprehensively adjust for health-care environment, which could have affected the relationship between multimorbidity and COPD medication receipt. Limitations include: a lack of generalizability due to inclusion of beneficiaries with fee-for-service claims only, restriction of Medicaid data to 4 states, and exclusion of dual Medicare/Medicaid 


\section{Multimorbidity ANd COPD MedicAtion Receipt}

enrollees. Due to the unavailability of laboratory data, we were not able to control for COPD severity or stage of COPD diagnosis, which can be an important covariate when assessing the relationship between multimorbidity and COPD medication receipt. Additionally, COPD diagnosis was based on ICD-9-CM diagnosis codes, which may not be the most accurate method to identify COPD. However, the validity of ICD-9-CM codes in identifying COPD has been established in prior studies. ${ }^{22,23}$ Our study was also limited by the use of diagnosis codes to identify depression. Although prior studies have used ICD-9-CM codes to identify depression, evidence exists that using diagnosis codes within claims data may lead to underestimation of depression. ${ }^{38}$

\section{Conclusions}

Despite these limitations, to the best of our knowledge, this is the first study to extensively examine the relationship between multimorbidity and COPD medication receipt. Our findings highlight the high prevalence of multimorbidity in non-elderly low-income adults with newly diagnosed COPD and poor COPD medication management in these subjects. Future research needs to examine provider factors, such as provider knowledge and experience in managing patients with multimorbidity, and patient factors, such as preferences and attitudes that may affect COPD management in the presence of multimorbidity.

\section{REFERENCES}

1. Boyd CM, Ritchie CS, Tipton EF, Studenski SA, Wieland D. From bedside to bench: summary from the American Geriatrics Society /National Institute on Aging research conference on comorbidity and multiple morbidity in older adults. Aging Clin Exp Res 2008;20(3): 181-188.

2. Fortin M, Soubhi H, Hudon C, Bayliss EA, van den Akker M. Multimorbidity's many challenges. BMJ 2007;334(7602):1016-1017.

3. Valderas JM, Starfield B, Sibbald B, Salisbury C, Roland M. Defining comorbidity: implications for understanding health and health services. Ann Fam Med 2009;7(4):357-363.

4. van den Akker M, Buntinx F, Knottnerus JA. Comorbidity or multimorbidity what's in a name? A review of literature. Eur J Gen Pract 1996;2(2):65-70.

5. Agency for Healthcare Research and Quality. Optimizing prevention and healthcare management for the complex patient. Updated 2007. http://archive.ahrq.gov/fund/trans101507.htm. Accessed June 10, 2014.

6. Marengoni A, Angleman S, Melis R, Mangialasche F, Karp A, Garmen A, et al. Aging with multimorbidity: a systematic review of the literature. Ageing Res Rev 2011;10(4):430-439.

7. Naessens JM, Stroebel RJ, Finnie DM, Shah ND, Wagie AE, Litchy WJ, et al. Effect of multiple chronic conditions among working-age adults. Am J Manag Care 2011;17(2):118-122.

8. Taylor AW, Price K, Gill TK, Adams R, Pilkington R, Carrangis N, et al. Multimorbidity—not just an older person's issue. Results from an Australian biomedical study. BMC Public Health 2010;10:718718.
9. Schnell K, Weiss CO, Lee T, Krishnan JA, Leff B, Wolff JL, Boyd C. The prevalence of clinically-relevant comorbid conditions in patients with physician-diagnosed COPD: a cross-sectional study using data from NHANES 1999-2008. BMC Pulm Med 2012;12:26.

10. Global Initiative for Chronic Lung Diseases. Global strategy for the diagnosis, management, and prevention of chronic obstructive pulmonary disease. Updated 2014. http://www.goldcopd.org/guidelinesglobal-strategy-for-diagnosis-management.html. Accessed August 10, 2014.

11. Han MK. Update in chronic obstructive pulmonary disease in 2010. Am J Respir Crit Care Med 2011;183(10):1311-1315.

12. Tsiligianni IG, Kosmas E, Van der Molen T, Tzanakis N. Managing comorbidity in COPD: a difficult task. Curr Drug Targets 2013; 14(2):158-176.

13. van der Woude HJ, Zaagsma J, Postma DS, Winter TH, van Hulst M, Aalbers R. Detrimental effects of beta-blockers in COPD: a concern for nonselective beta-blockers. Chest 2005;127(3):818-824.

14. Caughey GE, Preiss AK, Vitry AI, Gilbert AL, Roughead EE. Comorbid diabetes and COPD: impact of corticosteroid use on diabetes complications. Diabetes Care 2013;36(10):3009-3014

15. Suissa S, Kezouh A, Ernst P. Inhaled corticosteroids and the risks of diabetes onset and progression. Am J Med 2010;123(11):1001-1006.

16. Ogawa R, Stachnik JM, Echizen H. Clinical pharmacokinetics of drugs in patients with heart failure: an update (part 1, drugs administered intravenously). Clin Pharmacokinet 2013;52(3):169-185.

17. Kaiser Family Foundation. Medicaid matters: understanding Medicaid's role in our health care system. Publication 8165. Updated 2011. https://kaiserfamilyfoundation.files.wordpress.com/2013/01/8165.pdf. Accessed August 17, 2014.

18. Barnett K, Mercer SW, Norbury M, Watt G, Wyke S, Guthrie B. Epidemiology of multimorbidity and implications for health care, research, and medical education: a cross-sectional study. Lancet 2012; 380(9836):37-43.

19. Savva, G. Multimorbidity in the older population. http://www. thehealthwell.info/node/111238. Accessed August 10, 2014.

20. Dalal AA, Shah M, D'Souza AO, Chaudhari S, Crater G. Clinical and economic outcomes for patients initiating fluticasone propionate /salmeterol combination therapy $(250 / 50 \mathrm{mcg})$ versus anticholinergics in a comorbid COPD/depression population. Int $\mathrm{J}$ Chron Obstruct Pulmon Dis 2012;7:11-19.

21. Halpern R, Baker CL, Su J, Woodruff KB, Paulose-Ram R, Porter V, Shah H. Outcomes associated with initiation of tiotropium or fluticasone/salmeterol in patients with chronic obstructive pulmonary disease. Patient Prefer Adherence 2011;5:375-388.

22. Cooke CR, Joo MJ, Anderson SM, Lee TA, Udris EM, Johnson E, $\mathrm{Au} \mathrm{DH}$. The validity of using ICD-9 codes and pharmacy records to identify patients with chronic obstructive pulmonary disease. BMC Health Serv Res 2011;11:37.

23. Gershon AS, Wang C, Guan J, Vasilevska-Ristovska J, Cicutto L, To T. Identifying patients with physician diagnosed COPD in health administrative databases. COPD 2009;6(5):388-394.

24. Rabe KF, Hurd S, Anzueto A, Barnes PJ, Buist SA, Calverley P, et al. Global strategy for the diagnosis, management, and prevention of chronic obstructive pulmonary disease: GOLD executive summary. Am J Respir Crit Care Med 2007;176(6):532-555.

25. Fabbri LM, Luppi F, Beghé B, Rabe KF. Complex chronic comorbidities of COPD. Eur Respir J 2008;31(1):204-212.

26. Barnes PJ, Celli BR. Systemic manifestations and comorbidities of COPD. Eur Respir J 2009;33(5):1165-1185.

27. Nussbaumer-Ochsner Y, Rabe KF. Systemic manifestations of COPD. Chest 2011;139(1):165-173.

28. Goodman RA, Posner SF, Huang ES, Parekh AK, Koh HK. Defining and measuring chronic conditions: imperatives for research, policy, program, and practice. Prev Chronic Dis 2013;10:120239. 


\section{Multimorbidity AND COPD MedicAtion ReCeiPT}

29. Andersen RM. Revisiting the behavioral model and access to medical care: does it matter? J Health Soc Behav 1995;36(1):1-10.

30. Babitsch B, Gohl D, von Lengerke T. Re-revisiting Andersen's behavioral model of health services use: a systematic review of studies from 1998-2011. Psychosoc Med 2012;9:Doc11.

31. Lin PJ, Shaya FT, Scharf SM. Economic implications of comorbid conditions among Medicaid beneficiaries with COPD. Respir Med 2010;104(5):697-704

32. Au DH, Udris EM, Curtis JR, McDonell MB, Fihn SD, ACQUIP Investigators. Association between chronic heart failure and inhaled beta-2-adrenoceptor agonists. Am Heart J 2004;148(5):915920.

33. Lemaitre RN, Siscovick DS, Psaty BM, Pearce RM, Raghunathan TE, Whitsel EA, et al. Inhaled beta-2 adrenergic receptor agonists and primary cardiac arrest. Am J Med 2002;113(9):711-716.
34. Macie C, Wooldrage K, Manfreda J, Anthonisen N. Cardiovascular morbidity and the use of inhaled bronchodilators. Int $\mathrm{J}$ Chron $\mathrm{Ob}$ struct Pulmon Dis 2008;3(1):163-169.

35. Martin RM, Dunn NR, Freemantle SN, Mann RD. Risk of non-fatal cardiac failure and ischaemic heart disease with long acting beta 2 agonists. Thorax 1998;53(7):558-562.

36. Slatore CG, Bryson CL, Au DH. The association of inhaled corticosteroid use with serum glucose concentration in a large cohort. Am J Med 2009;122(5):472-478.

37. Ansari S, Hosseinzadeh H, Dennis S, Zwar N. Patients' perspectives on the impact of a new COPD diagnosis in the face of multimorbidity: a qualitative study. NPJ Prim Care Respir Med 2014;24:14036.

38. Noyes K, Liu H, Lyness JM, Friedman B. Medicare beneficiaries with depression: comparing diagnoses in claims data with the results of screening. Psychiatr Serv 2011;62(10):1159-1166. 